$\begin{array}{ccc}\text { Gazi University } & \text { Journal of Science } \\ \text { http://dergipark.gov.tr/gujs } & \text { (ged }\end{array}$

\title{
A new DoE-MTOPSIS based prediction model suggestion to capture potential SARS-CoV-2 reactivated patients
}

\author{
Yusuf Tansel IC * \\ Baskent University, Department of Industrial Engineering, 06810, Ankara, Turkey
}

\section{Highlights}

- Possibly available model is developed to predict a potential reactivated COVID-19 patient.

- A modified TOPSIS integrated DoE method is used to predict a potential reactivated COVID-19 patient.

- The presented novel model may be of interest to the readers studying similar research areas.

\begin{tabular}{l} 
Article Info \\
\hline Received: 24 June 2020 \\
Accepted: 16 Feb 2021 \\
Keywords \\
\hline SARS-CoV-2 \\
COVID-19 \\
Laboratory medicine \\
Design of experiment \\
TOPSIS meta-model
\end{tabular}

\section{INTRODUCTION}

A human-centred coronavirus (COVID-19) which is a new strain of RNA viruses was emerged in Wuhan/China, in December 2019. This virus is officially specified "Severe Acute Respiratory Syndrome Coronavirus-2 (SARS-CoV-2)" [1-2]. COVID-19 spread quickly across Asia-Pacific, Europe, Australia, USA, and other regions of the world. SARS-CoV-2 could induce different symptoms namely lymphopenia, dry cough, fever, fatigue, and dyspnoea in patients, and might result even death [1].

In the literature, Cheng and Shan [3] consolidated the epidemiology, clinical strategies, and virology of COVID-19 from both Chinese and English literature, and official documents. Furthermore, by fitting the number of infections with a single-term exponential model, they reported that the COVID-19 infection has been spread at an exponential rate, with a doubling period of 1.8 days. Another study titled "Clinical characteristics of severe acute respiratory syndrome coronavirus 2 reactivation"' by Ye et al. [1] aimed to test the clinical characteristics of the SARS-CoV-2 reactivation.

There are two main challenges associated with the pandemic that has spread around the world: (1) effectively controlling current and future outbreaks (2) effectively and safely treating infected patients. These issues should be evaluating before an emergency outbreak reach a critical infection threshold [4].

\begin{abstract}
Difficulties to use convenient data during the Severe Acute Respiratory Syndrome Coronavirus2 (SARS-CoV-2) pandemic outbreak and complexities of the problem attitude crucial challenges reactivated SARS-CoV-2 (COVID-19), we suggest a prediction model that beyond the clinical characteristics based evaluation approaches. In particular, we developed a possibly available and patient. Our paper aims to explore the applicability of a modified Technique for Order Preference by Similarity to Ideal Solutions (MTOPSIS) integrated Design of Experiment (DoE) method to The presented novel model may be of interest to the readers studying similar research areas. We illustrate MTOPSIS integrated DoE method by applying it to the COVID-19 pandemic real clinical cases from Wuhan/China-based data. Despite the small sample size, our study provides
an encouraging preliminary model framework. Finally, a step by step algorithm is suggested in the study for future research perspectives.
\end{abstract}


When evaluating based on countries, it is difficult to have the time and capacity to reach the figures to prove "what kind of treatment is effective for different segments of society during the pandemic?". In this case, the development of models and tools that can help physicians in the face of the difficulty of making "proper diagnosis" for different segments of society [5].

Due to the rapid spread of the SARS-CoV-2 epidemic in different parts of countries, more and more cases are becoming infected and raising the question of "whether negative cases are truly negative?" [6]. This issue was brought to the attention of the South Korean public on April 13. Accordingly, 116 COVID-19 cases that survived the disease were reported to be positive again [7]. Authorities say that further action is needed to ensure that a patient is recovering from COVID-19 or unable to reactivate after recovery [8]. So, given the importance of the COVID-19 global public health emergency, it is important to develop methodologies to identify patients with reactivation potential [1].

In the epidemiology literature, Prytherch et al. [9] developed an early warning system (EWS) for detecting patient deterioration. Meylan et al. [10] developed a EWS-based tool with data from 36 PCR-positive COVID-19 patients and used it in the early identification of COVID-19 patients requiring hospitalization in intensive care units (ICUs). The limited data sets suggested that the EWS could help clinicians predict COVID-19 patients who need to be sustained to the ICU.

In other studies, Overton et al. [11] proposed a tool of statistical approach beyond the simple differential equation applications for analysing the early stages of COVID-19 pandemic. Ogundokun et al. [12] presented the least-squares estimator to measure the impact of travel history on the spread of COVID-19 in Nigeria. Dawoud [13] used an autoregressive integrated moving average (ARIMA) method for predicting the COVID-19 cases in Palestine. Wang [14] investigated the Crow-Army Material Systems Analysis Activity (C-AMSAA) method to predict the COVID-19 indications by using daily issued data from the USA.

There is a need for systematically scientific tools to help the clinical decision-makers that can consider an activated possible COVID-19 patient. This paper aims to present the applicability of a modified Technique for Order Preference by Similarity to Ideal Solutions (MTOPSIS) integrated Design of Experiments (DoE) method to predict potential activated COVID-19 patients. In the literature, the TOPSIS-DoE approach is used for the first time by Ic [15] to select computer integrated manufacturing (CIM) technologies. The model is used to solve problems in banking and finance [16], production research environments [17-19], and financial performance measurement [20]. A key restriction of the TOPSIS-DoE model is the assignment of replicated importance scores of variables by decision-makers. Since each replication process needs a different decision-maker, a typical TOPSIS-DoE model needs a 3 to 5 decision-maker. Assigning 3 to 5 decision-maker may not be meaningful in COVID-19 syndrome that determining weights randomly might not be a suitable way to reach appropriate solutions. So, our study proposed the "assigning weights equally for all variables" approach by modifying the TOPSIS [21] application steps. The developed modified TOPSIS-DoE (MTOPSIS-DoE) approach's results compared with the ones obtained with the clinical symptoms. The author is not aware of any literature that predicts or captures potential SARS-CoV2 reactivated patients.

The paper is organized as follows: In Section 2, the methodology-based steps of the model are presented. In Section 3, case study results are proposed. In Section 4, the conclusions and future research perspectives are presented.

\section{MODIFIED TOPSIS-DOE METHOD}

This section presents the application steps of the MTOPSIS-DoE model (Figure 1) [15]. The clinical test results are suitable for the MTOPSIS-DoE model, one of the two restricted borders (low and high as input). The decision matrix $(\mathrm{R})$ includes a number of the clinical variables that lead to the development of an experimental design (Equation (1)). 
$R=\left\|r_{i j}\right\|$,

$(i=1, \ldots, m ; j=1, \ldots, n)$ of the $i$-th experiment for the $j$-th laboratory characteristics.

This design does not include the replication process of the experiment combination. The equally assigned importance scores for the clinical variables are normalized by dividing each importance score by the sum of the importance scores. The normalized decision matrix (A) (see Equation (1) and Equation (2) is transformed into a weighted normalized decision matrix (V) by using the Equations (3-6)

$$
\begin{aligned}
& \mathrm{A}=\left\|a_{i j}\right\| \\
& a_{i j}=\frac{r_{i j}}{\sqrt{\sum_{i=1}^{m} r_{i j}^{2}}} \\
& V=\left(\begin{array}{ccc}
w_{1} r_{11} & \cdots & w_{5} r_{15} \\
\vdots & \ddots & \vdots \\
w_{1} r_{321} & \cdots & w_{5} r_{325}
\end{array}\right) \\
& \sum_{j=1}^{n} w_{j}=1 \\
& \mathrm{~V}=\left(\begin{array}{ccc}
v_{11} & \cdots & v_{15} \\
\vdots & \ddots & \vdots \\
v_{321} & \cdots & v_{325}
\end{array}\right) .
\end{aligned}
$$

Finally, a unique ideal solution (UIS) is obtained with the application of the modified TOPSIS approach (Equation (7)):

$$
\overline{U I S}_{i}=\sqrt{\sum_{j=1}^{n}\left(v_{i j}-\overline{v_{J}^{*}}\right)^{2}}
$$

This solution is a ranking score for each potential reactivated COVID-19 patient. This ranking score, UIS, takes values between 0 and 1 .

Using the MTOPSIS-DoE results the polynomial regression equation (MTOPSIS meta-model) is determined (Equation (8)):

$$
\begin{aligned}
& Y=\beta_{0}+\sum_{i=1}^{k} \beta_{i} x_{i}+\sum_{i} \sum_{\substack{j \\
i<j}} \beta_{i j} x_{i} x_{j}+\sum_{i} \sum_{\substack{j \\
i<j<k}} \sum_{k} \beta_{i j k} x_{i} x_{j} x_{k} \\
& +\sum_{i} \sum_{\substack{j \\
i<j<k<l}} \sum_{k} \sum_{l} \beta_{i j k l} x_{i} x_{j} x_{k} x_{l}+\sum_{i} \sum_{\substack{j \\
i<j<k<l<m}} \sum_{k} \sum_{l} \sum_{m} \beta_{i j k l m} x_{i} x_{j} x_{k} x_{l} x_{m}+ \\
& \beta_{123456} x_{1} x_{2} x_{3} x_{4} x_{5} x_{6}+\varepsilon .
\end{aligned}
$$




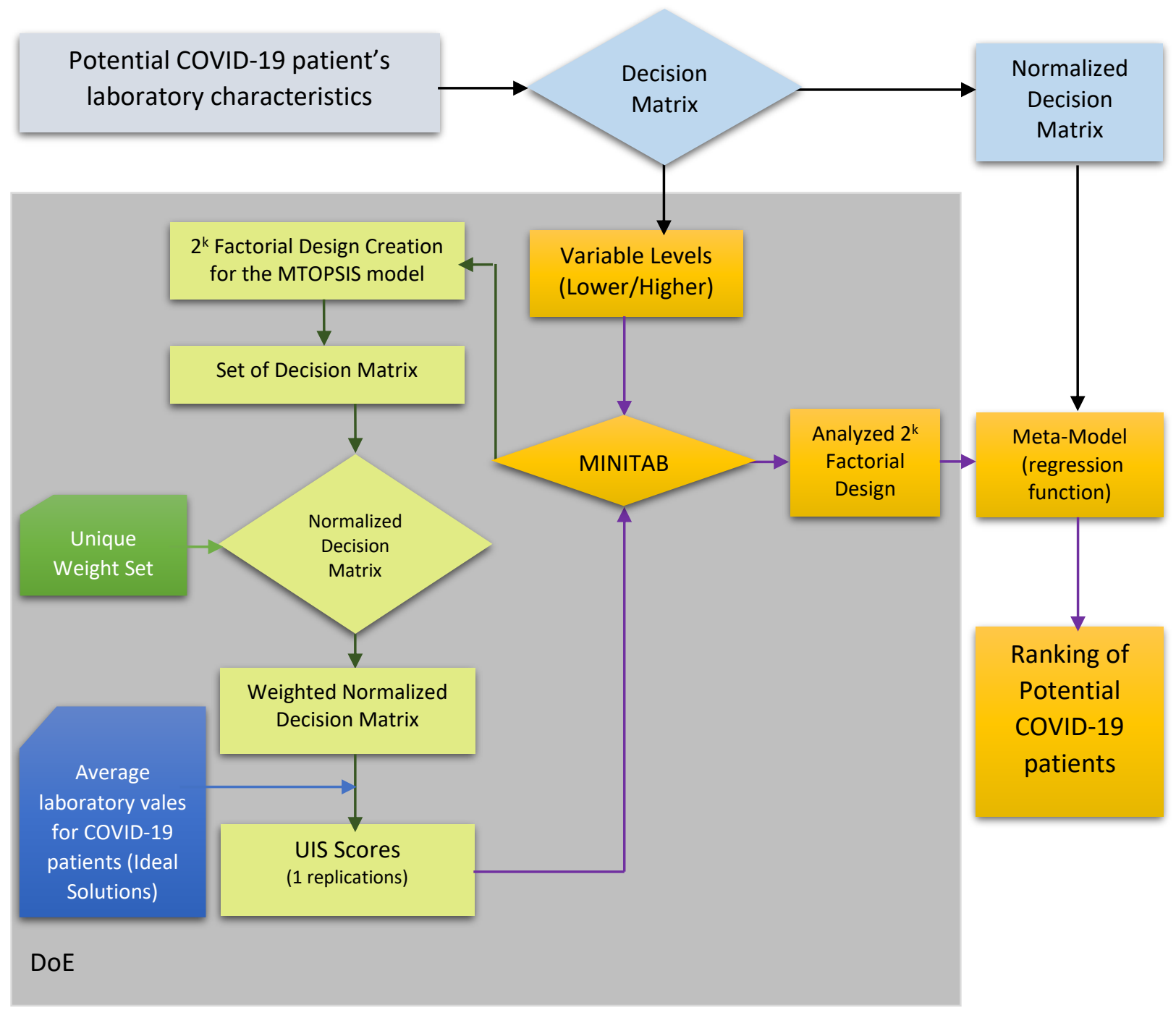

Figure 1. The MTOPSIS-DoE application steps

\section{CASE STUDY TO PREDICT A POTENTIAL REACTIVATED COVID-19 PATIENT}

Since it is not possible to use different data from different countries in the study, it is very crucial to use a unique data set from a single region and a specific period in terms of the suitability of the prediction results. There are very few studies and related data in the literature that can be used for our study. A data set suitable for use in our study is available in the study presented by Ye et al.'s [1] and Yang et al.'s [22] studies. So, our case study consists of six quantitative laboratory characteristics and five reactivated patients' information from Ye et al.'s [1] study. These entire six laboratory characteristics are "nominal value is better" type characteristics. Now to illustrate the proposed procedure to predict a potential reactivated COVID-19 patient through MTOPSIS-DoE application, various steps of the methodology are described below:

\section{Step 1: Determination of the factor level.}

According to Ye et al.'s [1] study (Table 1$)$, white blood cell count $(\times 109$ cells per L) (A) with lowerlevel 4.4 and higher-level 7.1, Neutrophil count $(\times 109$ cells per L) (B) with lower-level 1.8 and higherlevel 4.5, Lymphocyte count $(\times 109$ cells per L) (C) with lower-level 1.7 and higher-level 1, C-reactive protein (mg/L) (D) with lower-level 0 (N/A) and higher-level 23.7, ALT (U/L), (E) with lower-level 9 and higher-level 16, and AST(U/L) (F) with lower-level 13 and higher-level 22 are determined to variable levels affecting to predict a potential COVID-19 patient. 
Table 1. The $2^{6}$ factorial designs for MTOPSIS-DoE application

\begin{tabular}{|c|c|c|c|c|c|c|c|c|c|c|c|c|c|c|c|c|c|c|c|}
\hline \multirow[b]{2}{*}{ Exp. } & \multicolumn{6}{|c|}{ Factor Levels } & \multicolumn{6}{|c|}{ Normalized Matrix } & \multicolumn{6}{|c|}{ Equally weighted Normalized Matrix } & \multirow{2}{*}{\begin{tabular}{|c}
$\overline{U I S}$ \\
Distance \\
From \\
Average \\
Values \\
(Y)
\end{tabular}} \\
\hline & A & B & C & D & $\mathbf{E}$ & $\mathbf{F}$ & A & B & C & D & $\mathbf{E}$ & $\mathbf{F}$ & A & B & C & D & $\mathbf{E}$ & $\mathbf{F}$ & \\
\hline 1 & 4.4 & 4.5 & 1.3 & 1.24 & 0 & 0.04 & 0.150 & 0.164 & 0.140 & 0.177 & 0.087 & 0.077 & 0.025 & 0.027 & 0.023 & 0.029 & 0.014 & 0.013 & 3.295 \\
\hline 2 & 7.1 & 1.8 & 1.7 & 1.24 & 0.13 & 0.04 & 0.093 & 0.164 & 0.140 & 0.000 & 0.154 & 0.077 & 0.016 & 0.027 & 0.023 & 0.000 & 0.026 & 0.013 & 1.996 \\
\hline 3 & 4.4 & 4.5 & 1.3 & 0.4 & 0 & 0.04 & 0.093 & 0.066 & 0.107 & 0.177 & 0.154 & 0.159 & 0.016 & 0.011 & 0.018 & 0.029 & 0.026 & 0.027 & 2.237 \\
\hline 4 & 7.1 & 4.5 & 1.3 & 1.24 & 0 & 0.02 & 0.093 & 0.066 & 0.140 & 0.177 & 0.154 & 0.077 & 0.016 & 0.011 & 0.023 & 0.029 & 0.026 & 0.013 & 2.501 \\
\hline 5 & 4.4 & 1.8 & 1.7 & 1.24 & 0 & 0.04 & 0.093 & 0.164 & 0.107 & 0.177 & \begin{tabular}{|l|}
0.087 \\
\end{tabular} & \begin{tabular}{|l}
0.077 \\
\end{tabular} & 0.016 & 0.027 & 0.018 & 0.029 & 0.014 & \begin{tabular}{|l|l}
0.013 \\
\end{tabular} & 3.101 \\
\hline 6 & 4.4 & 1.8 & 1.3 & 1.24 & 0.13 & 0.02 & 0.093 & 0.066 & 0.107 & $\begin{array}{l}0.177 \\
\end{array}$ & \begin{tabular}{|l|l|}
0.154 \\
\end{tabular} & \begin{tabular}{|l|l|}
0.077 \\
\end{tabular} & 0.016 & 0.011 & 0.018 & 0.029 & 0.026 & \begin{tabular}{|l|}
0.013 \\
\end{tabular} & 2.412 \\
\hline 7 & 4.4 & 4.5 & 1.3 & 0.4 & 0 & 0.02 & 0.150 & 0.164 & 0.140 & 0.000 & 0.087 & \begin{tabular}{|l|l|}
0.159 \\
\end{tabular} & 0.025 & 0.027 & 0.023 & 0.000 & 0.014 & 0.027 & 2.584 \\
\hline 8 & 4.4 & 4.5 & 1.3 & 1.24 & \begin{tabular}{|l|l|}
0.13 \\
\end{tabular} & 0.04 & 0.093 & 0.066 & 0.140 & 0.000 & 0.154 & 0.077 & 0.016 & 0.011 & 0.023 & 0.000 & 0.026 & 0.013 & 1.699 \\
\hline 9 & 7.1 & 4.5 & 1.3 & 1.24 & 0 & 0.04 & 0.150 & 0.164 & 0.140 & 0.177 & 0.087 & 0.159 & 0.025 & 0.027 & 0.023 & 0.029 & 0.014 & 0.027 & 3.169 \\
\hline 10 & 4.4 & 4.5 & 1.3 & 1.24 & 0 & 0.02 & 0.093 & 0.066 & 0.107 & 0.000 & \begin{tabular}{|l|l|}
0.154 \\
\end{tabular} & 0.077 & 0.016 & 0.011 & 0.018 & 0.000 & 0.026 & \begin{tabular}{|l|}
0.013 \\
\end{tabular} & 1.564 \\
\hline 11 & 7.1 & 1.8 & 1.3 & 1.24 & 0.13 & 0.02 & 0.150 & 0.164 & 0.140 & 0.177 & 0.154 & 0.077 & 0.025 & 0.027 & 0.023 & 0.029 & 0.026 & \begin{tabular}{|l|l}
0.013 \\
\end{tabular} & 2.855 \\
\hline 12 & 7.1 & 1.8 & 1.7 & 0.4 & 0.13 & 0.04 & 0.150 & 0.164 & 0.107 & 0.000 & \begin{tabular}{|l|l|}
0.087 \\
\end{tabular} & \begin{tabular}{|l|l}
0.077 \\
\end{tabular} & 0.025 & 0.027 & 0.018 & 0.000 & 0.014 & \begin{tabular}{|l|l}
0.013 \\
\end{tabular} & 2.655 \\
\hline 13 & 7.1 & 4.5 & 1.3 & 0.4 & 0 & 0.04 & 0.150 & 0.066 & 0.107 & 0.000 & 0.087 & 0.159 & 0.025 & 0.011 & 0.018 & 0.000 & 0.014 & 0.027 & 2.267 \\
\hline 14 & 7.1 & 4.5 & 1.7 & 1.24 & 0.13 & 0.04 & 0.093 & 0.066 & 0.107 & 0.000 & 0.087 & 0.077 & 0.016 & 0.011 & 0.018 & 0.00 & 0.0 & 0.013 & 2.270 \\
\hline$\frac{14}{15}$ & 7.1 & 4.5 & 1.7 & $\frac{1.24}{1.24}$ & 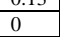 & $\frac{0.04}{0.04}$ & 0.093 & 0.0606 & 0.140 & 0.000 & $\begin{array}{l}0.001 \\
0.087\end{array}$ & 0.077 & $\frac{0.010}{0.016}$ & 0.011 & 0.0210 & 0.000 & 0.014 & 0.013 & 2.364 \\
\hline 16 & 4.4 & 4.5 & 1.7 & 0.4 & 0.13 & 0.04 & 0.093 & 0.066 & 0.140 & 0.177 & \begin{tabular}{|l|l|}
0.087 \\
\end{tabular} & \begin{tabular}{|l|l}
0.077 \\
\end{tabular} & 0.016 & 0.011 & 0.023 & 0.029 & 0.014 & \begin{tabular}{|l|l|}
0.013 \\
\end{tabular} & 2.993 \\
\hline 17 & 7.1 & 4.5 & 1.7 & 0.4 & 0.13 & 0.04 & 0.093 & 0.066 & 0.140 & 0.177 & 0.154 & \begin{tabular}{|l|l}
0.159 \\
\end{tabular} & 0.016 & 0.011 & 0.023 & 0.029 & 0.026 & \begin{tabular}{|l|l}
0.027 \\
\end{tabular} & 2.333 \\
\hline$\frac{17}{18}$ & 7.1 & 4.5 & 1.1 & 0.4 & $\begin{array}{l}0.13 \\
0.13\end{array}$ & $\frac{0.04}{0.04}$ & 0.093 & 0.160 & \begin{tabular}{|l|l|l|l|}
0.140 \\
\end{tabular} & 0.177 & $\begin{array}{l}0.154 \\
0.154\end{array}$ & \begin{tabular}{|l|l|l}
0.159 \\
\end{tabular} & $\frac{0.010}{0.016}$ & 0.021 & 0.023 & 0.029 & 0.026 & \begin{tabular}{|l}
0.027 \\
\end{tabular} & 2.558 \\
\hline 19 & 4.4 & 4.5 & 1.7 & 1.24 & 0 & 0.04 & 0.150 & 0.066 & 0.140 & 0.177 & 0.154 & \begin{tabular}{|l|l}
0.159 \\
\end{tabular} & 0.025 & 0.011 & 0.023 & 0.029 & 0.026 & 0.027 & 2.499 \\
\hline 20 & 4.4 & 1.8 & 1.3 & 1.24 & 0 & 0.04 & 0.150 & 0.066 & 0.140 & 0.000 & 0.087 & 0.077 & 0.025 & 0.011 & 0.023 & 0.000 & 0.014 & 0.013 & 2.528 \\
\hline$\frac{20}{21}$ & $\begin{array}{l}7.4 \\
7.1\end{array}$ & $\frac{1.0}{4.5}$ & $\begin{array}{l}1.3 \\
.3\end{array}$ & $\frac{1.24}{0.4}$ & 0 & $\frac{0.02}{0.02}$ & $\frac{1.150}{0.150}$ & 0.160 & $\frac{0.140}{0.140}$ & 0.000 & \begin{tabular}{|l|}
0.0151 \\
\end{tabular} & 0.159 & 0.025 & $\frac{0.011}{0.027}$ & 0.023 & 0.000 & 0.026 & 0.027 & $\begin{array}{l}.020 \\
1.993\end{array}$ \\
\hline 22 & 4.4 & 1.8 & 1.7 & 0.4 & 0.13 & 0.02 & 0.150 & 0.066 & 0.107 & 0.177 & \begin{tabular}{|l|}
0.154 \\
\end{tabular} & \begin{tabular}{|l|l|}
0.159 \\
\end{tabular} & 0.025 & 0.011 & 0.018 & 0.029 & 0.026 & \begin{tabular}{|l|l|}
0.027 \\
\end{tabular} & 2.409 \\
\hline 23 & 4.4 & 1.8 & 1.7 & 0.4 & 0 & 0.02 & 0.150 & 0.066 & 0.107 & 0.000 & \begin{tabular}{|l}
0.154 \\
\end{tabular} & \begin{tabular}{|l|l}
0.077 \\
\end{tabular} & 0.025 & 0.011 & 0.018 & 0.000 & 0.026 & 0.013 & 1.802 \\
\hline$\frac{3}{24}$ & $\begin{array}{l}7.4 \\
7.1\end{array}$ & $\frac{1.0}{4.5}$ & $\begin{array}{l}1.1 \\
.3\end{array}$ & $\frac{0.4}{1.24}$ & \begin{tabular}{|l|l}
0.13 \\
\end{tabular} & 0.04 & 0.150 & 0.160 & 0.140 & 0.177 & \begin{tabular}{|l|}
0.154 \\
0.087
\end{tabular} & \begin{tabular}{|l|l|}
0.077 \\
\end{tabular} & $\frac{0.023}{0.016}$ & 0.021 & 0.010 & 0.029 & 0.020 & 0.013 & $\begin{array}{l}3.002 \\
3.171\end{array}$ \\
\hline 25 & 4.4 & 1.8 & 1.3 & 0.4 & 0.13 & 0.02 & 0.093 & 0.066 & 0.107 & 0.000 & \begin{tabular}{|l}
0.154 \\
\end{tabular} & \begin{tabular}{|l}
0.159 \\
\end{tabular} & 0.016 & 0.011 & 0.018 & 0.000 & 0.026 & 0.027 & 1.278 \\
\hline 26 & 4.4 & 1.8 & 1.3 & 1.24 & 0 & 0.02 & 0.093 & 0.066 & 0.140 & 0.000 & 0.154 & 0.159 & 0.016 & 0.011 & 0.023 & 0.000 & 0.026 & \begin{tabular}{|l|}
0.027 \\
\end{tabular} & 1.440 \\
\hline 27 & 7.1 & 1.8 & 1.3 & 0.4 & 0 & 0.04 & 0.093 & 0.164 & 0.107 & 0.177 & \begin{tabular}{|l|l|}
0.087 \\
\end{tabular} & 0.159 & 0.016 & 0.027 & 0.018 & 0.029 & 0.014 & \begin{tabular}{|l|l|}
0.027 \\
\end{tabular} & 2.967 \\
\hline 28 & 4.4 & 4.5 & 1.7 & 0.4 & 0 & 0.04 & 0.150 & 0.164 & 0.107 & 0.177 & \begin{tabular}{|l|}
0.087 \\
\end{tabular} & \begin{tabular}{|l|l|}
0.159 \\
\end{tabular} & 0.025 & 0.027 & 0.018 & 0.029 & 0.014 & 0.027 & 3.099 \\
\hline 29 & 4.4 & 4.5 & 1.3 & 0.4 & 0.13 & 0.04 & 0.093 & 0.164 & 0.107 & 0.000 & \begin{tabular}{|l|}
0.154 \\
\end{tabular} & \begin{tabular}{|l|l|}
0.077 \\
\end{tabular} & 0.016 & 0.027 & 0.018 & 0.000 & 0.026 & 0.013 & 1.882 \\
\hline 30 & 7.1 & 4.5 & 1.7 & 1.24 & 0 & 0.02 & 0.150 & 0.066 & 0.140 & 0.000 & 0.087 & \begin{tabular}{|l|l|}
0.159 \\
\end{tabular} & 0.025 & 0.011 & 0.023 & 0.000 & 0.014 & 0.027 & 2.362 \\
\hline 31 & 7.1 & 1.8 & 1.7 & 1.24 & 0.13 & 0.02 & 0.150 & 0.066 & 0.140 & 0.000 & \begin{tabular}{|l|}
0.154 \\
\end{tabular} & 0.159 & 0.025 & 0.011 & 0.023 & 0.000 & 0.026 & 0.027 & 1.695 \\
\hline 32 & 4.4 & 1.8 & 1.7 & 0.4 & 0.13 & \begin{tabular}{|l|l|}
0.04 \\
\end{tabular} & 0.150 & 0.164 & 0.140 & 0.000 & 0.154 & 0.077 & 0.025 & 0.027 & 0.023 & 0.000 & 0.026 & 0.013 & 2.187 \\
\hline 33 & 7.1 & 4.5 & 1.7 & 0.4 & 0 & 0.02 & 0.093 & 0.066 & 0.140 & 0.000 & 0.087 & 0.159 & 0.016 & 0.011 & 0.023 & 0.000 & 0.014 & 0.027 & 2.186 \\
\hline 34 & 7.1 & 4.5 & 1.7 & 0.4 & 0 & 0.04 & 0.150 & 0.066 & 0.140 & 0.177 & \begin{tabular}{|l|}
0.154 \\
\end{tabular} & 0.077 & 0.025 & 0.011 & 0.023 & 0.029 & 0.026 & 0.013 & 2.656 \\
\hline 35 & 4.4 & $\begin{array}{ll}1.8 \\
1.8\end{array}$ & 1.3 & 0.4 & 0.13 & $\begin{array}{l}0.04 \\
0.04\end{array}$ & 0.093 & 0.164 & $\begin{array}{l}0.140 \\
0.107\end{array}$ & 0.000 & $\begin{array}{l}0.154 \\
\end{array}$ & 0.159 & 0.016 & 0.027 & 0.018 & 0.000 & 0.026 & 0.027 & $\begin{array}{l}1.050 \\
1.653\end{array}$ \\
\hline 36 & 7.1 & 4.5 & 1.3 & 1.24 & 0.13 & 0.02 & 0.150 & 0.066 & 0.107 & 0.000 & 0.154 & 0.159 & 0.025 & 0.011 & 0.018 & 0.000 & 0.026 & 0.027 & 1.560 \\
\hline 37 & 4.4 & 1.8 & 1.3 & 0.4 & 0 & 0.02 & 0.093 & 0.164 & 0.140 & 0.000 & 0.087 & 0.159 & 0.016 & 0.027 & 0.0 & 0.00 & 0.0 & 0.027 & 24 \\
\hline 38 & $\begin{array}{l}7.4 \\
7.1\end{array}$ & 1.0 & 1.3 & 0.4 & 0 & $\begin{array}{l}0.02 \\
0.02\end{array}$ & 0.150 & 0.164 & 0.1107 & 0.177 & 0.154 & $\begin{array}{l}0.0757 \\
0.077\end{array}$ & 0.025 & $\begin{array}{l}0.027 \\
0.027\end{array}$ & 0.018 & 0.029 & 0.026 & 0.017 & 2.4777 \\
\hline 39 & 7.1 & 1.8 & 1.3 & 1.24 & 0.13 & 0.04 & 0.093 & 0.164 & 0.107 & 0.000 & 0.087 & 0.077 & 0.016 & 0.027 & 0.018 & 0.000 & 0.014 & 0.013 & 2.500 \\
\hline 40 & 7.1 & 1.8 & 1.3 & 0.4 & 0.13 & 0.02 & 0.150 & 0.066 & 0.107 & 0.177 & 0.154 & 0.077 & 0.025 & 0.011 & 0.018 & 0.029 & 0.026 & 0.013 & 2.572 \\
\hline$\frac{40}{41}$ & 4.4 & 4.5 & 1.7 & $\frac{0.4}{1.24}$ & 0.13 & $\begin{array}{l}0.04 \\
0.04\end{array}$ & 0.150 & 0.164 & 0.107 & 0.000 & 0 & 0.077 & 0.025 & 0.027 & 0.0 & 0.000 & 0.026 & 0.013 & 2.084 \\
\hline 42 & 7.1 & 1.8 & 1.3 & 1.24 & 0 & 0.02 & 0.150 & 0.066 & 0.107 & 0.177 & 0.087 & 0.077 & 0.025 & 0.0 & & & 0.014 & 0.013 & 3.0 \\
\hline 43 & 4.4 & 4.5 & 1.7 & 1.24 & 0 & 0.02 & 0.150 & 0.066 & 0.140 & 0.177 & 0.087 & 0.077 & & 0.0 & & & & 0.013 & \\
\hline$\frac{43}{44}$ & $\begin{array}{l}7.4 \\
7.1\end{array}$ & 4 & $\begin{array}{l}.1 \\
1.7\end{array}$ & $\frac{1.24}{1.24}$ & \begin{tabular}{|l|}
0.13 \\
\end{tabular} & \begin{tabular}{ll|}
0.02 \\
\end{tabular} & 0.093 & 0.164 & $\frac{0.140}{0.140}$ & 0.000 & $\mid 0.087$ & 0.077 & $\frac{0.023}{0.016}$ & 0.027 & 0.023 & 0.000 & 0.014 & 0.013 & $\frac{3.124}{2.586}$ \\
\hline 45 & 4.4 & 1.8 & 1.7 & 0.4 & 0 & 0.04 & 0.150 & 0.164 & 0.140 & 0.177 & 0.154 & 0.159 & 0.025 & 0.027 & 0.023 & 0.029 & 0.026 & 0.027 & 2.709 \\
\hline 46 & 4.4 & 1.8 & 1.7 & 1.24 & 0.13 & 0.04 & 0.150 & 0.066 & 0.140 & 0.000 & 0.154 & 0.077 & 0.025 & 0.011 & & & & \begin{tabular}{|l|}
0.013 \\
\end{tabular} & 1.920 \\
\hline$\frac{40}{47}$ & $\begin{array}{l}4.4 \\
4.4\end{array}$ & $\begin{array}{l}.6 \\
1.8\end{array}$ & 1.1 & 0.44 & 0 & 0.04 & $\frac{0.150}{0.150}$ & 0.164 & $\frac{0.140}{0.107}$ & 0.000 & $\frac{0.154}{0.154}$ & 0.159 & 0.025 & 0.027 & $\frac{0.0}{0.0}$ & 0.000 & 0.026 & 0.027 & 1.879 \\
\hline 48 & 7.1 & 1.8 & 1.3 & 1.24 & 0 & 0.04 & 0.093 & 0.164 & 0.107 & 0.177 & 0.154 & 0.159 & 0.016 & 0.0 & 0.0 & 0.029 & 0.026 & 0.027 & 2.4 \\
\hline 49 & 7.1 & 4.5 & 1.7 & 0.4 & 0.13 & 0.02 & T. & 0.164 & & 0.000 & & & & & & & & & \\
\hline 50 & 4.4 & $\frac{4.5}{4.5}$ & 1.1 & $\frac{0.4}{0.4}$ & $\begin{array}{l}0.13 \\
0.13\end{array}$ & $\frac{0.02}{0.02}$ & $\frac{0.093}{0.150}$ & 0.1044 & 0.107 & 0.177 & \begin{tabular}{|l|}
0.08 \\
0.154 \\
\end{tabular} & $\frac{0.159}{0.159}$ & $\frac{0.010}{0.025}$ & $\begin{array}{l}0.027 \\
0.027\end{array}$ & 0.0108 & 0.000 & 0.014 & 0.027 & $\frac{2.352}{2.627}$ \\
\hline 51 & 4.4 & 1.8 & 1.7 & 1.24 & 0.13 & 0.02 & 0.093 & 0.16 & 0.1 & 0.177 & & $\overline{0.0}$ & & 0.0 & $\overline{0.0}$ & 0.029 & $\overline{0.0}$ & 0.013 & 2.629 \\
\hline 52 & 7.1 & 1.8 & 1.7 & 1.24 & 0 & & 0.150 & & & 0.177 & & & & & & & & \begin{tabular}{|l|l|}
0.013 \\
\end{tabular} & \\
\hline 53 & 7.1 & 1.8 & 1.7 & 0.4 & 0 & 0.02 & $\begin{array}{l}0.150 \\
0.150\end{array}$ & 0.164 & 0.107 & 0.000 & \begin{tabular}{|l|}
0.087 \\
\end{tabular} & 0.159 & 0.025 & 0.027 & 0.018 & 0.000 & 0.014 & 0.027 & 2.497 \\
\hline 54 & 4.4 & 1.8 & 1.3 & 1.24 & 0.13 & \begin{tabular}{l|l|}
0.04 \\
\end{tabular} & 0.093 & 0.066 & 0.107 & 0.177 & 0.087 & 0.077 & 0.016 & 0.011 & 0.0 & 0.029 & 0.014 & 0.013 & 2.919 \\
\hline 55 & 7.1 & 1.8 & 1.3 & 0.4 & 0.13 & 0.04 & 0.150 & 0.06 & 0.107 & 0.177 & 0.0 & 0.1 & 0.0 & & & 0.02 & & 0.027 & 2.9 \\
\hline 56 & 4.4 & 4.5 & 1.3 & 1.24 & 0.13 & 0.02 & 0.093 & 0.164 & 0.1 & 0.177 & 0.1 & & & 0.0 & & 0.029 & 0.0 & \begin{tabular}{|l|l|}
0.013 \\
\end{tabular} & 2.712 \\
\hline 57 & 7.1 & 1.8 & 1.7 & 0.4 & 0.13 & 0.02 & $\begin{array}{l}0.093 \\
\end{array}$ & 0.164 & 0.140 & 0.177 & & & & 0.0 & & & & \begin{tabular}{|l|}
0.027 \\
\end{tabular} & \\
\hline 58 & 7.1 & 4.5 & 1.3 & 0.4 & 0.13 & $\begin{array}{l}0.02 \\
0.02 \\
\end{array}$ & $\begin{array}{l}0.0150 \\
0.150\end{array}$ & 0.164 & $\frac{0.140}{0.140}$ & 0.000 & \begin{tabular}{|l|}
0.087 \\
0.087
\end{tabular} & \begin{tabular}{|l|}
0.077 \\
\end{tabular} & 0.025 & $\begin{array}{l}0.027 \\
0.027\end{array}$ & 0.023 & 0.000 & 0.014 & \begin{tabular}{|l|}
0.013 \\
\end{tabular} & $\frac{2.041}{2.736}$ \\
\hline 59 & 7.1 & 1.8 & 1.7 & 1.24 & 0 & 0.02 & 0.093 & 0.066 & 0.107 & 0.177 & 0.087 & 0.159 & 0.016 & 0.011 & \begin{tabular}{|l|l|}
0.018 \\
\end{tabular} & \begin{tabular}{|l|l|}
0.029 \\
\end{tabular} & 0.014 & \begin{tabular}{|l|l|}
0.027 \\
\end{tabular} & 2.776 \\
\hline 60 & 7.1 & 1.8 & 1.7 & 0.4 & T & 0.04 & 0.093 & 0.066 & 0.107 & 0.000 & & 0.159 & & & & & & \begin{tabular}{|l|l|}
0007 \\
\end{tabular} & \\
\hline 61 & 4.4 & 4.5 & 1.7 & 0.4 & 0 & 0.02 & 0.093 & 0.066 & 0.140 & 0.177 & & $\frac{0.1579}{0.159}$ & 0.0 .1 & & & & & 0.027 & 2.8 \\
\hline 62 & 4.4 & 1.8 & 1.7 & 1.24 & 0 & 0.02 & 0.150 & 0.066 & 0.107 & 0.000 & & & & 0.0 & & 000 & 0.014 & \begin{tabular}{|l|l|}
0.013 \\
\end{tabular} & 2.4 \\
\hline 63 & 4.4 & 4.5 & 1.7 & 1.24 & 0.13 & 0.02 & 0.150 & 0.066 & 0.140 & 0.177 & \begin{tabular}{|l|}
0.087 \\
\end{tabular} & \begin{tabular}{|l|l|}
0.159 \\
\end{tabular} & 0.025 & 0.011 & 0.023 & 0.029 & 0.014 & \begin{tabular}{|l|}
0.027 \\
\end{tabular} & 2.991 \\
\hline 64 & 4.4 & 4.5 & 1.3 & 0.4 & 0.13 & 0.02 & 0.093 & 0.164 & 0.140 & 0.000 & \begin{tabular}{|l|}
0.154 \\
\end{tabular} & \begin{tabular}{|l|}
0.159 \\
\end{tabular} & 0.016 & 0.027 & 0.023 & 0.000 & 0.026 & \begin{tabular}{|l|l|}
0.027 \\
\end{tabular} & 1.781 \\
\hline & & & & & & & & & & & & & & & & & & & \\
\hline$=\frac{r_{i j}}{\sqrt{\sum_{i=1}^{m} r_{i j}^{2}}}$ & 47 & 27 & 12 & 134 & 104 & 170 & & & & & 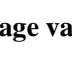 & lues ${ }^{a}$ & 4.56 & 2.6 & 1.21 & 7.25 & 20 & 23 & \\
\hline & & & & & & & & & $i_{7}$ & ave & age & lues & 0.0161 & 0.0158 & 0.0167 & 0.0090 & 0.0321 & 0.0226 & \\
\hline
\end{tabular}

a From Yang et al.'s [22] study: Clinical characteristics of the 2019 novel coronavirus disease (COVID-19):A multi-center study in Wenzhou city, Zhejiang, China. Sex: Male/Female (81/68). ${ }^{1}$

${ }^{b} \sqrt{ }\left(4.4^{2}+7.1^{2}+\ldots+4.4^{2}+4.4^{2}\right)=47.3$.

\footnotetext{
${ }^{1}$ Yang et al. [22] states that: "...A total of 149 patients, including 40 patients from Wenzhou central hospital, 64 patients from Ruian people's hospital and 45 patients from Yueqing people's hospital were recruited. The average age was $45.11 \pm 13.35$ years old, and 81 patients (54.4\%) were males. A total of 85 patients had Hubei travel/residence history, 49 patients had contact with people from Hubei province and 15 patients had no traceable exposure history to Hubei. Of the 149 patients, 52 (34.9\%) had underlying chronic diseases, including cerebrovascular diseases, digestive diseases, etc...."
} 


\section{Step 2: Experimental design.}

The variables (factors) are the independent parameters that are used as inputs to the MTOPSIS model to obtain the dependent variable (Y). Table 1 shows the "combination matrix" (decision matrix for TOPSIS) for 26 factorial design (64 combinations) with outputs (Y: MTOPSIS scores) by using the equal weights for each laboratory characteristics (factors/criteria). The results (Y) are analysed by analysis of variance (ANOVA) procedures by using the MINITAB19 software (Tables 2 and 3). The obtained regression equation (Equation (9)) is as follows:

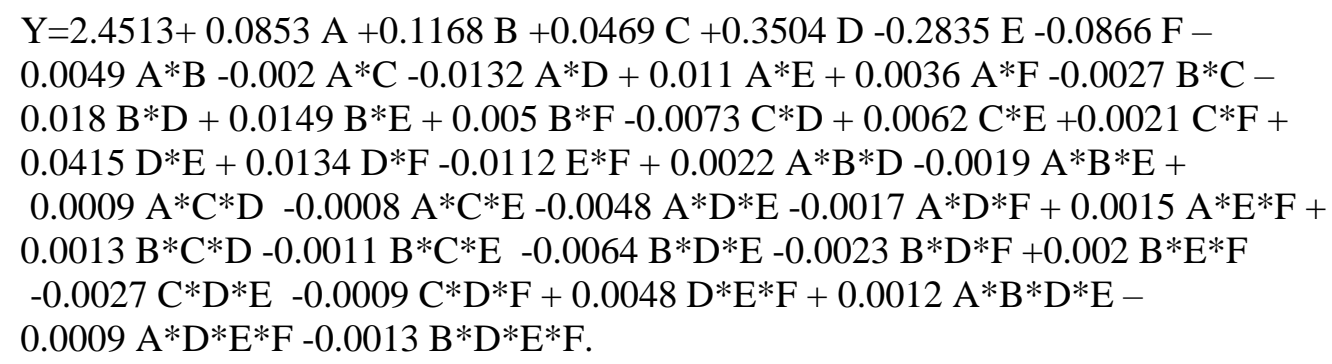

\section{Step 3: Calculations of the ranking scores for possible reactivated patients.}

Patients had no underlying diseases such as diabetes, hypertension or cardiovascular disease [1]. On the other hand, all of the patients excluded influenza virus infection upon admission to hospital. We can use the meta-model (Equation (9)) to predict the possible patients (Figure 2). As the outcome of the model, it can be stated that the third patient is the closest patient to the average results given by Yang et al. [22]. On contrary, the first patient is the farthest patient to the average results given by Yang et al. [22]. Using the developed model, the score for a new potential patient to be calculated with his/her laboratory values. Calculated scores can give an idea of whether he/she is a potential patient or not. Clinical characteristics are supported our results: Four of the 5 patients except for patient 4, presented with a fever without chills. Patient 4 showed normal body temperature [1]. Among the male patients, (Patient 1 and 2) patient 1 has better clinical characteristics than patient 2. Patient 1 had not cough, had not sore throat, and had not dyspnoea according to the Ye et al. [1]'s report. On the other hand, among the female patients, (Patient 3,4, and 5) patient 4 has the best clinical characteristics than the others (patient 3, and 5). Patient 4 had not cough, had not sore throat, had not fever, and had not dyspnoea according to the Ye et al. [1]'s report (Table 4).

\section{CONCLUSION}

In this study, we have suggested a new MTOPSIS-DoE prediction model on the data of the reactivated confirmed cases of COVID-19 in Wuan/China. The developed model in our study is open to development concerning other possible pandemics in the future. So, the more laboratory characteristics (factors) and their confirmed data can easily include in the developed model.

Although our study was restricted by the small sample of patients from Ye et al.[1]'s study, we believe that the proposed model is useful to predict the laboratory results and SARS-CoV-2 potential in COVID-19 patients. As a result of the limited published literature about the reactivation process for the COVID-19, the used data set is limited $(\mathrm{N}=5)$. Briefly studying the epidemiological literature revealed that the reactivation cases of COVID-19 do not sufficiently confirm, but might have been false positives (actually, this is what will be studied in later studies). Given the importance of this disease, we probably may not present confirmed models that are $100 \%$ scientifically sound by using the data here is thin. However, we can propose the scientific way appropriate for future studies using the big data-related and confirmed for the COVID-19 reactivation process. Our study presents a simple framework model related to "how MTOPSIS-DoE model can use for the capture to the reactivated SARS-CoV-2 potentials in COVID-19 patients". 
Table 2. Estimated effects and coefficients for $Y$

\begin{tabular}{|c|c|c|c|c|c|}
\hline Term & Effect & Coef & SE Coef & $\mathrm{T}$ & $\mathrm{P}$ \\
\hline Constant & & 2.4513 & 0.000063 & 39220 & 0 \\
\hline A & 0.1705 & 0.0853 & 0.000063 & 1364 & 0 \\
\hline B & 0.2336 & 0.1168 & 0.000063 & 1868.5 & 0 \\
\hline $\mathrm{C}$ & 0.0939 & 0.0469 & 0.000063 & 751 & 0.001 \\
\hline D & 0.7009 & 0.3504 & 0.000063 & 5607 & 0 \\
\hline $\mathbf{E}$ & -0.5669 & -0.2835 & 0.000063 & -4535.5 & 0 \\
\hline $\mathbf{F}$ & -0.1731 & -0.0866 & 0.000063 & -1385 & 0 \\
\hline $\mathbf{A}^{* \mathbf{B}}$ & -0.0098 & -0.0049 & 0.000063 & -78.5 & 0.008 \\
\hline $\mathrm{A} * \mathrm{C}$ & -0.004 & -0.002 & 0.000063 & -32 & 0.02 \\
\hline $\mathbf{A} * \mathbf{D}$ & -0.0264 & -0.0132 & 0.000063 & -211 & 0.003 \\
\hline $\mathbf{A} * \mathbf{E}$ & 0.0219 & 0.011 & 0.000063 & 175.5 & 0.004 \\
\hline $\mathbf{A} * \mathbf{F}$ & 0.0072 & 0.0036 & 0.000063 & 58 & 0.011 \\
\hline $\mathrm{B} * \mathrm{C}$ & -0.0053 & -0.0027 & 0.000063 & -42.5 & 0.015 \\
\hline B*D & -0.0359 & -0.018 & 0.000063 & -287.5 & 0.002 \\
\hline $\mathbf{B} * \mathbf{E}$ & 0.0299 & 0.0149 & 0.000063 & 239 & 0.003 \\
\hline $\mathbf{B} * \mathbf{F}$ & 0.0099 & 0.005 & 0.000063 & 79.5 & 0.008 \\
\hline C*D & -0.0146 & -0.0073 & 0.000063 & -117 & 0.005 \\
\hline $\mathbf{C} * \mathbf{E}$ & 0.0123 & 0.0062 & 0.000063 & 98.5 & 0.006 \\
\hline $\mathbf{C} * \mathbf{F}$ & 0.0041 & 0.0021 & 0.000063 & 33 & 0.019 \\
\hline $\mathbf{D} * \mathbf{E}$ & 0.0831 & 0.0415 & 0.000063 & 664.5 & 0.001 \\
\hline $\mathbf{D} * \mathbf{F}$ & 0.0268 & 0.0134 & 0.000063 & 214 & 0.003 \\
\hline $\mathbf{E} * \mathbf{F}$ & -0.0223 & -0.0112 & 0.000063 & -178.5 & 0.004 \\
\hline $\mathrm{A} * \mathrm{~B} * \mathrm{C}$ & 0.0007 & 0.0003 & 0.000063 & 5.5 & 0.114 \\
\hline A*B*D & 0.0044 & 0.0022 & 0.000063 & 35.5 & 0.018 \\
\hline $\mathbf{A} * \mathbf{B} * \mathbf{E}$ & -0.0039 & -0.0019 & 0.000063 & -31 & 0.021 \\
\hline $\mathrm{A} * \mathrm{~B} * \mathrm{~F}$ & -0.0016 & -0.0008 & 0.000063 & -12.5 & 0.051 \\
\hline$A * C * D$ & 0.0017 & 0.0009 & 0.000063 & 14 & 0.045 \\
\hline $\mathbf{A} * \mathbf{C} * \mathbf{E}$ & -0.0017 & -0.0008 & 0.000063 & -13.5 & 0.047 \\
\hline $\mathrm{A} * \mathrm{C} * \mathrm{~F}$ & -0.0006 & -0.0003 & 0.000063 & -5 & 0.126 \\
\hline $\mathbf{A} * \mathbf{D} * \mathbf{E}$ & -0.0096 & -0.0048 & 0.000063 & -76.5 & 0.008 \\
\hline $\mathbf{A} * \mathbf{D} * \mathbf{F}$ & -0.0034 & -0.0017 & 0.000063 & -27 & 0.024 \\
\hline $\mathbf{A} * \mathbf{E} * \mathbf{F}$ & 0.0029 & 0.0015 & 0.000063 & 23.5 & 0.027 \\
\hline B*C*D & 0.0026 & 0.0013 & 0.000063 & 20.5 & 0.031 \\
\hline$B * C * E$ & -0.0021 & -0.0011 & 0.000063 & -17 & 0.037 \\
\hline $\mathrm{B} * \mathrm{C} * \mathrm{~F}$ & -0.0008 & -0.0004 & 0.000063 & -6.5 & 0.097 \\
\hline B*D*E & -0.0127 & -0.0064 & 0.000063 & -102 & 0.006 \\
\hline B*D*F & -0.0046 & -0.0023 & 0.000063 & -36.5 & 0.017 \\
\hline B*E*F & 0.004 & 0.002 & 0.000063 & 32 & 0.02 \\
\hline$C * D * E$ & -0.0053 & -0.0027 & 0.000063 & -42.5 & 0.015 \\
\hline $\mathbf{C} * \mathbf{D} * \mathbf{F}$ & -0.0019 & -0.0009 & 0.000063 & -15 & 0.042 \\
\hline $\mathrm{C}^{*} \mathrm{E}^{*} \mathrm{~F}$ & 0.0016 & 0.0008 & 0.000063 & 12.5 & 0.051 \\
\hline $\mathbf{D}^{*} \mathbf{E}^{*} \mathbf{F}$ & 0.0097 & 0.0048 & 0.000063 & 77.5 & 0.008 \\
\hline $\mathrm{A} * \mathrm{~B} * \mathrm{C} * \mathrm{D}$ & -0.0007 & -0.0003 & 0.000063 & -5.5 & 0.114 \\
\hline $\mathrm{A} * \mathrm{~B} * \mathrm{C} * \mathrm{E}$ & 0.0005 & 0.0003 & 0.000063 & 4 & 0.156 \\
\hline $\mathrm{A} * \mathrm{~B} * \mathrm{C} * \mathrm{~F}$ & 0.0003 & 0.0002 & 0.000063 & 2.5 & 0.242 \\
\hline $\mathbf{A} * \mathbf{B} * \mathbf{D} * \mathbf{E}$ & 0.0025 & 0.0012 & 0.000063 & 20 & 0.032 \\
\hline $\mathrm{A} * \mathrm{~B} * \mathrm{D} * \mathrm{~F}$ & 0.0009 & 0.0005 & 0.000063 & 7.5 & 0.084 \\
\hline $\mathrm{A}^{*} \mathrm{~B} * \mathrm{E} * \mathrm{~F}$ & -0.0009 & -0.0004 & 0.000063 & -7 & 0.09 \\
\hline $\mathrm{A}^{*} \mathrm{C} * \mathrm{D} * \mathrm{E}$ & 0.0012 & 0.0006 & 0.000063 & 9.5 & 0.067 \\
\hline$A * C * D * F$ & 0.0004 & 0.0002 & 0.000063 & 3 & 0.205 \\
\hline $\mathrm{A}^{*} \mathrm{C}^{*} \mathrm{E}^{*} \mathrm{~F}$ & -0.0003 & -0.0002 & 0.000063 & -2.5 & 0.242 \\
\hline $\mathbf{A} * \mathbf{D} * \mathbf{E} * \mathbf{F}$ & -0.0018 & -0.0009 & 0.000063 & -14.5 & 0.044 \\
\hline $\mathrm{B} * \mathrm{C} * \mathrm{D} * \mathrm{E}$ & 0.0014 & 0.0007 & 0.000063 & 11 & 0.058 \\
\hline $\mathrm{B} * \mathrm{C} * \mathrm{D} * \mathrm{~F}$ & 0.0006 & 0.0003 & 0.000063 & 4.5 & 0.139 \\
\hline $\mathrm{B} * \mathrm{C} * \mathrm{E} * \mathrm{~F}$ & -0.0006 & -0.0003 & 0.000063 & -5 & 0.126 \\
\hline $\mathbf{B} * \mathbf{D} * \mathbf{E} * \mathbf{F}$ & -0.0026 & -0.0013 & 0.000063 & -21 & 0.03 \\
\hline C*D*E*F & -0.0011 & -0.0005 & 0.000063 & -8.5 & 0.075 \\
\hline $\mathrm{A} * \mathrm{~B} * \mathrm{C} * \mathrm{D} * \mathrm{E}$ & -0.0005 & -0.0002 & 0.000063 & -4 & 0.156 \\
\hline $\mathrm{A} * \mathrm{~B} * \mathrm{C} * \mathrm{D} * \mathrm{~F}$ & -0.0003 & -0.0002 & 0.000063 & -2.5 & 0.242 \\
\hline $\mathrm{A} * \mathrm{~B} * \mathrm{C} * \mathrm{E} * \mathrm{~F}$ & 0.0001 & 0.0001 & 0.000063 & 1 & 0.5 \\
\hline $\mathrm{A} * \mathrm{~B} * \mathrm{D} * \mathrm{E} * \mathrm{~F}$ & 0.0008 & 0.0004 & 0.000063 & 6 & 0.105 \\
\hline $\mathrm{A}^{*} \mathrm{C} * \mathrm{D} * \mathrm{E}^{*} \mathrm{~F}$ & 0.0003 & 0.0002 & 0.000063 & 2.5 & 0.242 \\
\hline $\mathrm{B} * \mathrm{C} * \mathrm{D} * \mathrm{E} * \mathrm{~F}$ & 0.0004 & 0.0002 & 0.000063 & 3 & 0.205 \\
\hline
\end{tabular}


Table 3. Analysis of variance for $Y$

\begin{tabular}{|l|l|l|l|l|l|l|}
\hline Source & DF & Seq SS & Adj SS & Adj MS & F & P \\
\hline Main Effects & 6 & 14.9608 & 14.9608 & 2.49347 & 9973871 & 0 \\
\hline 2-Way Interactions & 15 & 0.1944 & 0.1944 & 0.01296 & 51831.6 & 0.003 \\
\hline 3-Way Interactions & 20 & 0.0079 & 0.0079 & 0.0004 & 1582.5 & 0.02 \\
\hline 4-Way Interactions & 15 & 0.0004 & 0.0004 & 0.00003 & 103.53 & 0.077 \\
\hline 5-Way Interactions & 6 & 0 & 0 & 0 & 12.42 & 0.214 \\
\hline Residual Error & 1 & 0 & 0 & 0 & & \\
\hline Total & 63 & 15.1635 & & & & \\
\hline S $=0.0005$ & R-Sq $=100.00 \%$ & R -Sq(adj) $=$ & $100.00 \%$ & & & \\
\hline
\end{tabular}

Table 4. Clinical characteristics for patients [1]

\begin{tabular}{|l|c|c|c|c|c|}
\hline Patient & 1 & 2 & 3 & 4 & 5 \\
\hline Sex & Male & Male & Female & Female & Female \\
\hline Age (years) & 30 & 42 & 32 & 27 & 31 \\
\hline SARS-CoV-2 negative to positive (days) & 4 & 8 & 17 & 15 & 9 \\
\hline Fatigue & $\sqrt{ }$ & $\sqrt{ }$ & $\sqrt{ }$ & $\sqrt{ }$ & $\sqrt{ }$ \\
\hline Typical signs of viral infection & $\sqrt{ }$ & $\sqrt{ }$ & $\sqrt{ }$ & $\sqrt{ }$ & $\sqrt{ }$ \\
\hline Dyspnoea & $\mathrm{X}$ & $\mathrm{X}$ & $\mathrm{X}$ & $\mathrm{X}$ & $\mathrm{X}$ \\
\hline Fever on admission & $\sqrt{ }$ & $\sqrt{ }$ & $\sqrt{ }$ & $\mathrm{X}$ & $\sqrt{ }$ \\
\hline Cough & $\mathrm{X}$ & $\sqrt{ }$ & $\mathrm{X}$ & $\mathrm{X}$ & $\mathrm{X}$ \\
\hline Sore throat & $\mathrm{X}$ & $\mathrm{X}$ & $\sqrt{ }$ & $\mathrm{X}$ & $\mathrm{X}$ \\
\hline
\end{tabular}

Despite the small sample size, our study provides an encouraging preliminary model framework. This study provides a proof-of-concept prediction model for potential reactivated SARS-CoV-2 (COVID-19) patients. Still, in time of the on-going pandemic outbreak, our simple model may demonstrate useful for initial triage and following monitoring of patients.

There are some complexities when developing a prediction model for potential reactivated SARS-CoV-2 (COVID-19) patients. We have proposed an integrated model to serve as part of a generally appropriate tool. But, the proposed MTOPSIS-DoE model is subject to some restrictions. An important one of these is the lack of homogenous population-based clinical data, such as through age-plication [23], and different risk-groups [24], and temporal or seasonal diversifications [25], all of which affect model predictions. Furthermore, the developed MTOPSIS-DoE model uses a unique and equal weight set for each criterion or factor. Nowadays, we do not sense which criteria are more important than the others in the diagnosis of the COVID-19 reactivation? If this question can clarify by the infection scientist, we use more appropriate methodologies such as the Analytic Hierarchy Process (AHP)/ Analytic Network Process (ANP) for the criteria weighting process. Finally, the relative simplicity of the presented MTOPSIS-DoE model yields for the improvement of qualitative intuition concerning the influence of different clinical procedures while obtaining tractable practical frameworks which can be further investigate and better inform policy-makers. As a result of this study, Figure 3 illustrates a step-by-step algorithm for future research perspectives. 


\begin{tabular}{|c|c|c|c|c|c|c|c|c|}
\hline \multirow{2}{*}{$\begin{array}{l}\text { Values for patients }[1] \\
\text { White blood cell count }(\times 109 \text { cells per } L)\end{array}$} & \multirow{2}{*}{$\begin{array}{c}\text { patient1 } \\
\mathbf{5 . 9} \\
\end{array}$} & \multirow{2}{*}{\begin{tabular}{|c|} 
paitent2 \\
7.1 \\
\end{tabular}} & patient3 & \multicolumn{2}{|c|}{ paitent 4} & \multicolumn{2}{|c|}{ patient5 } & aNormalization \\
\hline & & & 4.4 & & & 4.5 & & 47.3 \\
\hline Neutrophil count $(\times 109$ cells per $L)$ & 3.5 & 4.5 & 1.8 & & & 2.6 & & 27.4 \\
\hline Lymphocyte count $(\times 109$ cells per $\mathrm{L})$ & 1.7 & 1.3 & 1.7 & & & 1.4 & & 12.1 \\
\hline C-reactive protein $(\mathrm{mg} / \mathrm{L})$ & 18.7 & 23.7 & 0 & & & 0 & & 134.1 \\
\hline $\operatorname{ALT}(\mathrm{U} / \mathrm{L})$ & 40 & 16 & 11 & & & 10 & & 103.8 \\
\hline AST(U/L) & 32 & 19 & 20 & & & 22 & & 169.5 \\
\hline \multicolumn{9}{|l|}{ From Table 2} \\
\hline Normalized values for patients & patient1 & paitent 2 & \multicolumn{2}{|c|}{ patient3 } & \multicolumn{2}{|c|}{ paitent4 } & \multicolumn{2}{|c|}{ patient5 } \\
\hline White blood cell count $(\times 109$ cells per $L)$ & ${ }^{a} 0.124866$ & 0.150262 & \multicolumn{2}{|c|}{0.09312} & \multicolumn{2}{|c|}{0.137564} & \multicolumn{2}{|c|}{0.095236} \\
\hline Neutrophil count $(\times 109$ cells per $\mathrm{L})$ & 0.127659 & 0.164133 & \multicolumn{2}{|c|}{0.065653} & \multicolumn{2}{|c|}{0.149543} & 0.094832 & 94832 \\
\hline Basophile count $(\times 109$ cells per $\mathrm{L})$ & 0.079057 & 0.158114 & \multicolumn{2}{|c|}{0.118585} & \multicolumn{2}{|c|}{0.079057} & 0.079057 & 79057 \\
\hline C-reactive protein $(\mathrm{mg} / \mathrm{L})$ & 0.139482 & 0.176777 & \multicolumn{2}{|c|}{0} & \multicolumn{2}{|c|}{0} & \multicolumn{2}{|c|}{0} \\
\hline ALT (U/L) & 0.385186 & 0.154074 & \multicolumn{2}{|c|}{0.105926} & \multicolumn{2}{|c|}{0.086667} & 0.096296 & 96296 \\
\hline AST(U/L) & 0.188772 & 0.112083 & 0.117 & 982 & 0.07 & 6688 & 0.1 & 29781 \\
\hline \multicolumn{9}{|l|}{$5.9 / 47.3=0.124866$} \\
\hline \multicolumn{9}{|c|}{$\begin{array}{l}\mathbf{Y}_{1}=2.4513+(0.0853 * 0.124866)+(0.1168 * 0.150262)+(0.0469 * 0.09312)+(0.3504 * 0.137564)-(0.2835 * 0.095236)- \\
(0.0866 * 0.178621)-(0.0049 * 0.018763)-(0.002 * 0.011627)-(0.0132 * 0.017177)+(0.011 * 0.011892)+(0.0036 * 0.022304)- \\
(0.0027 * 0.013992)-(0.018 * 0.020671)+(0.0149 * 0.01431)+(0.005 * 0.02684)-(0.0073 * 0.01281)+(0.0062 * 0.008868)+ \\
(0.0021 * 0.016633)+(0.0415 * 0.013101)+(0.0134 * 0.024572)-(0.0112 * 0.017011)+(0.0022 * 0.002581)- \\
(0.0019 * 0.001787)+(0.0009 * 0.0016)-(0.0008 * 0.001107)-(0.0048 * 0.001636)-(0.0017 * 0.003068)+(0.0015 * 0.002124)+ \\
(0.0013 * 0.001925)-(0.0011 * 0.001333)-(0.0064 * 0.001969)-(0.0023 * 0.003692)+(0.002 * 0.002556)-(0.0027 * 0.00122)- \\
(0.0009 * 0.002288)+(0.0048 * 0.00234)+(0.0012 * 0.000246)-(0.0009 * 0.000292)-(0.0013 * 0.000352)=\mathbf{3 . 5 5 8}\end{array}$} \\
\hline
\end{tabular}

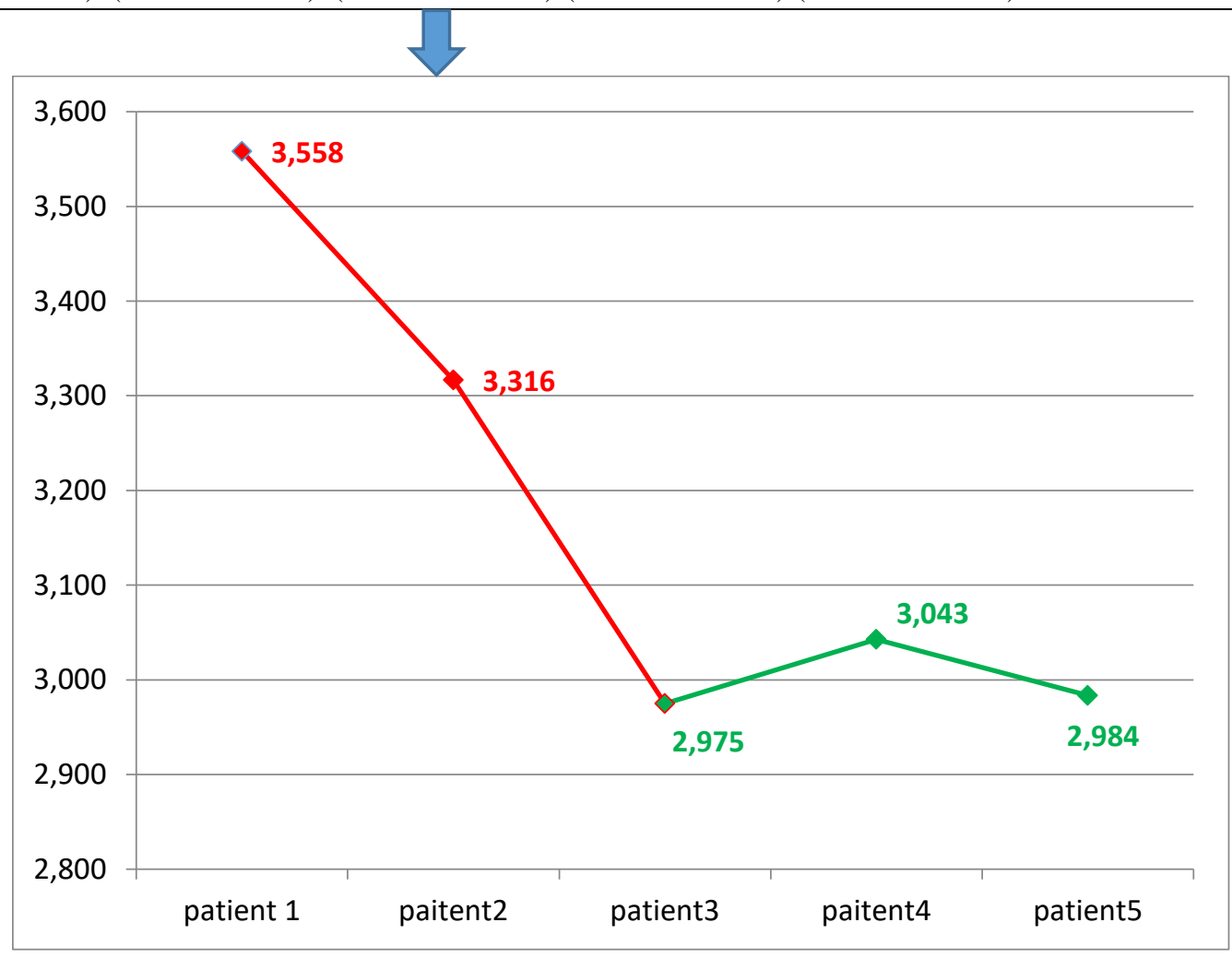

Figure 2. Calculation of MTOPSIS DoE-score for patients 


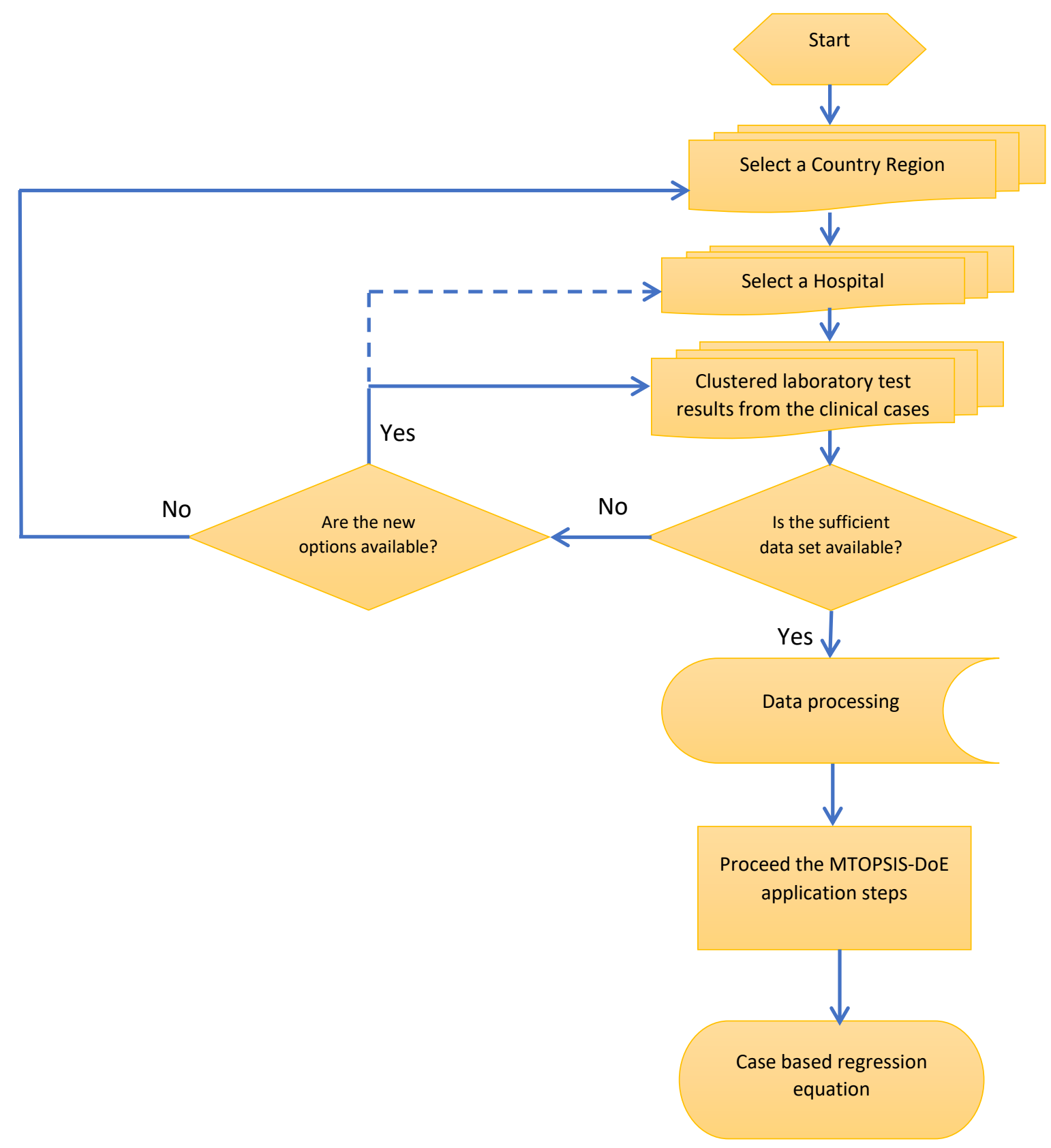

Figure 3. An algorithm for the future research perspective

\section{CONFLICT OF INTEREST}

No conflict of interest was declared by the author.

\section{REFERENCES}

[1] Ye, G., Pan, Z., Pan, Y., Deng, Q., Chen, L., Li, J., Li, Y., Wang, X., "Clinical characteristics of severe acute respiratory syndrome coronavirus 2 reactivation”, Journal of Infection, 80(5): e14-e17, (2020). 
[2] Zhu, Y., Chen, Y.Q., "On a statistical transmission model in analysis of the early phase of COVID-19 outbreak", Statistics in Biosciences, 13, 1-17, (2021). DOI: https://doi.org/10.1007/s12561-02009277-0.

[3] Cheng, Z.J., Shan, J., "2019 Novel coronavirus: where we are and what we know", Infection, 48: 155$163,(2020)$.

[4] Salzberger, B., Glück, T., Ehrenstein, B., "Successful containment of COVID-19: the WHO-report on the COVID-19 outbreak in China", Infection, 48: 151-153, (2020).

[5] Baraboutis, I.G., Gargalianos, P., Aggelonidou, E. Adraktas, A., "Initial real-life experience from a designated COVID-19 centre in Athens, Greece: A proposed therapeutic algorithm", SN Comprehensive Clinical Medicine, 2(6): 1-5, (2020).

[6] Alizargar, J., "Risk of reactivation or reinfection of novel coronavirus (COVID-19)", Journal of the Formosan Medical Association, 119(6): 1123, (2020).

[7] Smith, J., "South Korea reports more recovered coronavirus patients testing positive again", Available from: https://www.reuters.com/article/us-health-coronavirus-southkorea/south-korea-reports-morerecovered-coronavirus-patients-testing-positive-again-idUSKCN21V0JQ.6. Access date: 03/12/2020.

[8] Kayat, S., "Doctor's Note: Can the coronavirus reactivate?", Available from: https://www. aljazeera. com/indepth/features/doctor-note-coronavirus-reactivate-200412062905537.html. Access date: 04 / 12/2020.

[9] Prytherch, D.R., Smith, G.B., Schmidt, P.E., Featherstone, P.I., "ViEWS - Towards a national early warning score for detecting adult inpatient deterioration", Resuscitation, 81: 932-7, (2010).

[10] Meylan, S., Akrour, R., Regina, J., Bart, P.A., Dami, F., Calandra, T., "An early warning score to predict ICU admission in COVID-19 positive patients", Journal of Infection, 81(5): 816-846, (2020).

[11] Overton, C.E., Stage, H. B., Ahmad, S., Curran-Sebastian, J., Dark, P., Das, R., Fearon, E., Felton, T., Fyles, M., Gent, N., Hall, I., House, T., Lewkowicz, H., Pang, X., Pellis, L., Sawko, R., Ustianowski, A., Vekaria, B., Webb, L., "Using statistics and mathematical modelling to understand infectious disease outbreaks: COVID-19 as an example", Infectious Disease Modelling, 5: 409441,(2020).

[12] Ogundokun, R. O., Lukman, A.F., Kibria, G.B., Awotunde, J.B., Aladeitan, B.B., "Predictive modelling of COVID-19 confirmed cases in Nigeria", Infectious Disease Modelling, 5: 543-548, (2020).

[13] Dawoud, I., "Modelling Palestinian COVID-19 cumulative confirmed cases: A comparative study", Infectious Disease Modelling, 5: 748-754, (2020).

[14] Wang, Y., "Predict new cases of the coronavirus 19; in Michigan, USA or other countries using Crow-AMSAA method", Infectious Disease Modelling, 5: 459-477, (2020).

[15] Ic, Y.T., "An experimental design approach using TOPSIS method for the selection of computerintegrated manufacturing technologies", Robotics and Computer-Integrated Manufacturing, 28: 245256, (2012).

[16] Ic, Y.T., "A TOPSIS based design of experiment approach to assess company ranking", Applied Mathematics and Computation, 227: 630-647, (2014). 
[17] Ic, Y.T., "Development of a new multi-criteria optimization method for engineering design problems", Research in Engineering Design, 27(4): 413-436, (2016).

[18] Ic, Y.T., Yurdakul, M., "Understanding the effect of assignment of importance scores of evaluation criteria randomly in the application of DOE-TOPSIS in decision making", In: Advances in Intelligent Systems and Computing, Springer, 411-424, (2018).

[19] Chakraborty, S., Chatterjee, P., Das, P.P., "A DoE-TOPSIS method-based meta-model for parametric optimization of non-traditional machining processes", Journal of Modelling in Management, 14(2): 430-455, (2019).

[20] Bilbao-Terol, A., Arenas-Parra, M., Alvarez-Otero, S., Cañal-Fernández, V., "Integrating corporate social responsibility and financial performance", Management Decision, 57(2): 324-348, (2019).

[21] Ic, Y.T., Şimşek, E., "Operating window perspective integrated TOPSIS approach for hybrid electrical automobile selection”, SN Applied Sciences, 1(11): 1314, (2019).

[22] Yang, W., Cao, Q., Qin, L.E., Wang, X., Cheng, Z., Pan, A., Dai, J., Sun, Q., Zhao, F., Qu, Z., Yan, F., "Clinical characteristics and imaging manifestations of the 2019 novel coronavirus disease (COVID-19): A multi-centre study in Wenzhou city, Zhejiang, China", Journal of Infection, 80(4): 388-393, (2020).

[23] Pellis, L., Cauchemez, S., Ferguson, N.M., Fraser, C., "Systematic selection between age and household structure for models aimed at emerging epidemic predictions", Nature Communications, 11(1): 1-11, (2020).

[24] Valdano, E., Poletto, C., Boelle, P.Y., Colizza, V., "Reorganization of nurse scheduling reduces the risk of healthcare associated infections", medRxiv, 19007724, (2019).

[25] Lau, M.S., Dalziel, B.D., Funk, S., McClelland, A., Tiffany, A., Riley, S., Metcalf, C. J. E., Grenfell, B.T., "Spatial and temporal dynamics of super spreading events in the 2014-2015 West Africa Ebola epidemic", Proceedings of the National Academy of Sciences, 114(9): 2337-2342, (2017). 